\title{
La protection internationale des investissements en République démocratique du Congo à l'aune de l'affaire Patrick Mitchell devant le Centre International pour le Règlement des Différends relatifs aux Investissements (CIRDI)
}

\author{
Par Guy-Prosper DJUMA BILALI LOKEMA*
}

\section{Résumé en français}

Dans « La protection internationale des investissements en République démocratique du Congo à l'aune de l'affaire Patrick Mitchell devant le Centre International pour le Règlement des Différends relatifs aux Investissements (CIRDI) », l'auteur analyse principalement l'affaire Patrick Mitchell pour soutenir l'existence en République démocratique du Congo des voies judiciaires internationales efficaces qui assurent la protection des investissements. L'auteur précise que la faiblesse du système judiciaire congolais, miné par la corruption, la partialité des juges ainsi que le dysfonctionnement de l'appareil judiciaire, ne doit pas et ne peut pas constituer un frein aux investissements privés étrangers.

La protection de ces investissements est obtenue au plan international, outre par l'existence d'une procédure de cassation devant la Cour Commune de Justice et d'Arbitrage (Abidjan, Côte d'Ivoire) dans le cadre du droit Ohada, mais surtout par le CIRDI, sans omettre le fait que la Cour internationale de Justice (La Haye, Pays-Bas) peut également pourvoir à ladite protection judiciaire à travers le mécanisme de la protection diplomatique.

D'une complexité remarquable du fait de la mise en œuvre de trois étapes importantes d'une procédure devant un tribunal CIRDI allant de la sentence arbitrale du 9 février 2004 à l'annulation de celle-ci par un Comité ad hoc le $1^{\text {er }}$ novembre 2006 en passant par la suspension de l'exécution de la sentence le 30 novembre 2004, l'affaire Patrick Mitchell fixe l'attention. Elle permet de se rendre compte de la réalité de la protection des investissements dans un double aspect jouant en faveur de l'investisseur ou au bénéfice du pays hôte, plaidant ainsi pour une confiance mutuelle en l'arbitrage CIRDI. Aucun favoritisme n'est accordé, ni à l'investisseur étranger ni à la République démocratique du Congo.

\section{Résumé en anglais}

In « La protection internationale des investissements en République démocratique du Congo à l'aune de l'affaire Patrick Mitchell devant le Centre international pour le Règlement des Différends relatifs aux Investissements » (CIRDI) (International protection of in-

* Doctorant en droit public à l'Université de Kinshasa, Assistant à la Faculté de droit de l'Université de Kinshasa et Avocat au Barreau de Kinshasa/Gombe 
vestments in Democratic Republic of Congo on the basis of Patrick Mitchell case before the Centre for Settlement of Investment Disputes (ICSID), the author mainly analyses Patrick Mitchell case for supporting the existence in Democratic Republic of Congo of effective international judiciary ways which assure investments protection. The author specifies that the weakness of Congolese judiciary system, undermined by corruption, by judges'bias and by the dysfunction of judiciary apparatus, must not and cannot constitute a restraint to foreign private investments.

The protection of these investments is obtained in international level, beyond the existence of cassation procedure before the Common Court of Justice and Arbitration (Abidjan, Ivory Coast) in the framework of Harmonization of Business Law in Africa (Ohada), but especially by the International Centre for Settlement of Investment Disputes, without omitting the fact that International Court of Justice (The Hague, Netherlands) can also provide judiciary protection said through diplomatic protection mechanism.

Patrick Mitchell case fixes attention due to his remarkable complexity because of implementation of procedure three important steps before an ICSID Tribunal going from 9 February 2004 Award to the annulment of that Award, on the 1st November 2006 by Ad hoc Committee through the Award enforcement suspension on the 30 November 2004. This case allows to realize the reality of investments protection in a double aspect which plays in the direction of investor' favor or in the direction of host state' benefit. So, it pleads for mutual confidence in ICSID arbitration favor.

\section{Introduction}

Si l'existence des juridictions internationales et le recours de plus en plus fréquent à ces dernières sont des phénomènes relativement récents, l'idée d'une justice internationale est une réalité assez ancienne qui s'est de longue date mise en œuvre alors même que l'on ne songeait guère à un tribunal ou à toute autre juridiction internationale instituée et permanente $^{1}$. Très longtemps, en effet, la justice était rendue sur le plan international par l'arbitrage dont l'organisation, sous l'influence des divers principes westphaliens, et très respectueuse de l'égalité juridique et de la souveraineté des États, a facilité la mise en place et justifié les faveurs des souverains et des États.

Ainsi, au XIX siècle devait également voir d'assez nombreux arbitrages ad hoc pris par des souverains individuellement (le roi d'Espagne, d'Italie ou d'Angleterre, le Président de la République française ou des États-Unis) ou par des corps comme le sénat de Hambourg ou le conseil confédéral suisse. Cette renaissance de l'arbitrage au XIXème siècle a vu aussi son début de juridictionnalisation. ${ }^{2}$

1 Auguste Mampuya Kanunk'a Tshiabo, Les juridictions internationales ce qu'elles sont, ce qu'elles font?, Cours de Droit international public, Destiné aux apprenants en DES, Faculté de Droit Université de Kinshasa, 2011-2012, p.4.

2 Carreau Dominique, Droit international, Paris, 2004, p.608. 
Concomitamment à ces vérités, l'idée d'instituer une juridiction internationale permanente à vocation universelle ne s'est matérialisée qu'assez récemment avec la Cour permanente de Justice internationale, supplantée par la Cour internationale de Justice qui a forgée la tendance juridictionnelle du droit international. Cette maturation a conduit au renforcement d'une justice internationale dite transnationale car opposant les États aux personnes privées ressortissants des États. C'est sous l'inspiration de ces évidences que la Banque mondiale, butée à nombre des différends surgissant entre États et investisseurs ressortissants des États, a résolu d'initier une juridiction d'arbitrage entre les États et les privés. Ce qui aboutit, le 18 mars 1965 à Washington, à l'adoption entre quelques Etats occidentaux de la convention instituant le CIRDI.

La convention a été élaborée par les administrateurs de la Banque internationale pour la reconstruction et le développement. Le 18 mars 1965, les administrateurs ont soumis la convention, avec un rapport, à l'examen des gouvernements membres de la Banque Mondiale en vue de sa signature et de sa ratification. La convention est entrée en vigueur le 14 octobre 1966, suite à la ratification de vingt États. Au 10 avril 2006, 143 pays avaient ratifié la convention pour devenir des États contractants.

Sans s'égarer dans des conjectures, l'analyse du CIRDI se situe dans le cadre des relations transnationales qui mettent en présence les États et les citoyens d'autres États. A la différence de la pratique traditionnelle étatique qui impose aux individus de saisir les juridictions internationales à travers le mécanisme de la protection diplomatique ${ }^{3}$, avec tous les inconvénients ${ }^{4}$ que cette voie représente, la procédure devant le CIRDI est toute autre.

Le règlement des différends internationaux relatifs aux investissements par le canal du CIRDI échappe à la voie classique et assure une accessibilité directe des individus aux instances internationales. Ce mécanisme prend le contre-pied du procédé interétatique traditionnel puisque le particulier, personne physique ou morale, n'a pas besoin de suivre le tracé de l'endossement de son État national ou, même dans certains cas, sinon dans la majorité, de ne pas se soumettre à la règle générale de l'épuisement des voies de recours internes ${ }^{5}$.

3 La protection diplomatique est définie comme le fait pour un État de prendre fait et cause pour son national, victime de la violation du droit international par un autre État afin de faire cesser les violations du droit international.

4 La protection diplomatique est soumise à des conditions drastiques notamment celles liées à sa recevabilité à savoir la nationalité ainsi que l'épuisement des voies de recours internes. Ces exigences ne garantissent toujours pas l'aboutissement de cette voie. Il n'en demeure pas moins que la protection diplomatique constitue une attribution étatique que le Gouvernement exerce au gré de ses intérêts. L'abandon et l' " inopérabilité » de la clause calvo en disent long.

5 Selon l'article 26 de la convention de Washington du 18 mars 1965 relative au Règlement des Différends Internationaux relatifs aux Investissements entre les États et ressortissants d'un autre État « Le consentement des parties à l'arbitrage dans le cadre de la présente Convention est, sauf stipulation contraire, considéré comme impliquant renonciation à l'exercice de tout autre recours. Comme condition à son consentement à l'arbitrage dans le cadre de la présente Convention, un Etat contractant peut exiger que les recours administratifs ou judiciaires internes soient épuisés.». Sur la question de l'épuisement des voies de recours internes, on lira en droit international général notamment Bertrand Bauchot, La protection diplomatique des individus en droit international, Mémoire de 
Il n'est donc pas vain à ce stade de rappeler que l'institutionnalisation d'une juridiction internationale, quelle que soit sa forme, est un facteur récent des relations internationales puisque les États ont été, dès l'origine, opposés à l'idée de l'intervention d'un juge pour trancher les différends les opposant.

La désaffection des sujets primaires du droit international pour un tribunal international a été surmontée avec le recours à l'arbitrage international. Ce dernier aspect de règlement des litiges interétatiques a pris une ampleur particulière avec l'affaire de l'Alabama mettant aux prises les États-Unis à la Grande-Bretagne en marge de la guerre civile américaine ${ }^{6}$. Dès lors que les États eurent admis l'intervention des tiers dans leur contentieux selon diverses modalités ${ }^{7}$, il s'en est suivi la création et l'institutionnalisation des juridictions internationales judiciaires ou arbitrales $^{8}$, permanentes ${ }^{9}$ ou ad hoc $^{10}$.

Dans cette ruée vers la judiciarisation de la société internationale, une place s'est créée pour les différends relatifs aux investissements. Ces derniers sont véritablement à l'origine de la création du CIRDI. Il faut ajouter à cela le mauvais fonctionnement du système judiciaire des États du tiers-monde, notamment la partialité des juges et le déni de justice.

La question de la protection des investissements s'est posé avec acuité aux lendemains des indépendances des États sud-américains, asiatiques et africains. La pomme de discorde a été longtemps la possibilité ou non pour les États de procéder à la nationalisation des sociétés privées étrangères. La nationalisation des intérêts privés en droit interne a toujours

D.E.A, Université de Lille II, droit et santé, Faculté des sciences juridiques, politiques et sociales, 2001-2002; en jurisprudence, on consultera particulièrement, en ce qui concerne la Cour internationale de Justice, l'affaire Interhandel, Ahmadou Sadio Dialo. Dans la doctrine africaine, et une étude à paraître Djuma Bilali, « La règle de l'épuisement des voies de recours internes à la lumière de la jurisprudence de la Commission africaine des droits de l'homme et des peuples », Actes de la conférence internationale sur le 30th Anniversary on African Charter of Human and peolpe's Rights,5-7 novembre 2012, Unisa, Pretoria. Sur la jurisprudence africaine, en l'occurrence celle de la Commission africaine des droits de l'homme et des peuples, on consultera Communication 221/98, Afred B. Cudjoe c/Ghana; Communication 90/93, Paul S. Haye c/Gambie; Communication 18/88, El Hady Boubacar Diawara c/Benin; Communication 135/94, Kenya Human Rights Commission c/Kenya; Communication 60/91, Constitutional Rights Project c/Nigeria.

6 Sur l'affaire Alabama voir la sentence arbitrale du 14 septembre 1872.

7 La composition du tribunal arbitral est soit à arbitre unique, soit à trois ou à cinq avec dans chaque cas un surarbitre.

8 On signalera principalement la Cour permanente d'Arbitrage ainsi que les juridictions arbitrales ponctuelles qui pullulent à travers le globe.

9 Il existe aujourd'hui une multitude de tribunaux internationaux permanents selon l'angle géographique ou matériel. D'après l'aspect géographique, faisant application du critère de l'universalisme ou du régionalisme, on peut citer la Cour pénale internationale, le tribunal international du droit de la mer, l'organe de règlement de l'OMC, la Cour internationale de Justice.

10 Les juridictions ad hoc pullulent particulièrement dans le domaine répressif où nous avons le Tribunal pénal international pour l'ex-Yougoslavie institué en vertu de la Résolution 933 du Conseil de sécurité des Nations Unies, le tribunal pénal international pour le Rwanda sécrété par la Résolution 955 du Conseil de sécurité des Nations Unies. 
divisé la société en deux blocs, entre les pro nationalisations et les anti. Les politiques de gauche l'ont souvent favorisée à l'inverse des programmes de la droite.

On citera l'affaire du canal de Suez. Il y a lieu de rappeler que la crise du canal de Suez est consécutive à la nationalisation par Nasser de la compagnie privée qui exploitait le canal de Suez. A la suite de cette nationalisation, la France et l'Angleterre dont étaient originaires les actionnaires majoritaires de la compagnie précitée, et pour des raisons stratégiques liées au contrôle de la navigation sur le canal de Suez par où transite l'important trafic pétrolier vers le golfe Persique, vont entrer en guerre contre l'Égypte en 1956. Il a fallu la menace d'intervention de l'Union Soviétique pour que les armées françaises et britanniques cessent leur offensive ${ }^{11}$.

Pour parer à la tyrannie des États s'autorisant d'exproprier les biens privés sans indemnisation préalable, il a fallu mettre en place un mécanisme juridique et juridictionnel qui assure la protection des investisseurs. Si aujourd'hui l'expropriation est admise sur le plan international, assortie de la garantie d'une indemnisation juste et préalable, elle est néanmoins à l'origine de plusieurs litiges transnationaux.

La promotion ainsi que la protection des investissements étrangers revêtent une importance particulière pour les différents gouvernements établis de par le monde, et particulièrement dans les Etats du tiers-monde. Qu'il soit bien précisé que ces investissements ont un impact direct sur la croissance économique des Etats bénéficiaires. Ils assurent l'accroissement des capitaux, la résorption du chômage à travers la création de milliers d'emplois.

D'une importance qu'on ne peut plus camoufler, les investissements ne demeurent pas une équation à la fois pour les investisseurs qui doivent chercher à les protéger, et pour les gouvernements qui aspirent en accueillirent davantage comme gage de leur bonne gouvernance économique.

Relevant d'une importance capitale, la protection des investissements est un sujet majeur en République démocratique du Congo. L'assainissement du climat des affaires est l'une des préoccupations du gouvernement de la RDC pour attirer les investisseurs privés étrangers. C'est sous un argument, erroné et volontairement fauché d'attraction des investissements privés, que l'adhésion de la RDC à l'Ohada a été acquise.

Mettant en œuvre le libéralisme économique depuis la Conférence de Berlin et l'Acte qui en est sorti, modifié par la convention de Saint Germain-en-Laye, la République démocratique du Congo, à l'instar de tous les Etats du monde, est ouverte aux capitaux étrangers provenant de l'extérieur. L'immensité de ce pays aux dimensions continentales suffit d'attester de la difficulté de l'Etat de pouvoir développer seul le pays. Les ressources finan-

11 En République démocratique du Congo, on se souviendra de la spectaculaire nationalisation de l'Union Minière du Haut Katanga par le Président Mobutu en 1966. L'action de l'ancien maréchal du Zaïre n'est pas étrangère à la brouille diplomatique entre Kinshasa et Bruxelles. Les mesures de zaïrianisation, radicalisation et rétrocession de 1973, 1974 et 1975 interviennent dans un contexte international particulièrement marqué par un mouvement tendant à lutter contre les expropriations sauvages des biens privés, singulièrement étrangers. Au moment desdites mesures, la Convention de Washington du 18 mars 1965 est déjà en vigueur depuis le 14 octobre 1966. 
cières étant limitées, il est impossible d'assister à une omniprésence de l'Etat congolais sur le plan économique. S'il est défaillant dans les domaines qui relèvent de la puissance étatique, il ne faut pas le chercher dans le domaine économique dans lequel la tendance est à «moins d'Etat».

La valeur des investissements s'en ressent très fortement. L'Etat ne peut pas investir à la fois dans les transports maritime, fluvial, ferroviaire, routier, dans la métallurgie, l'agroforesterie, la biotechnologie, l'agropastorale, les industries de transformation, l'énergie, etc. C'est trop demander à l'Etat congolais dont les tentacules financiers sont extrêmement réduits. Nous n'entrons même pas dans les réalités de gestion financière de la cité congolaise.

Sans forcer, la main est donc tendue aux investisseurs tant nationaux qu'internationaux. Si les nationaux pourraient garder un certain silence coupable face au manque de protection de la part des juridictions locales, tel n'est pas le cas des investisseurs étrangers. Ces derniers n'hésitent pas à faire intervenir leurs Etats en cas de différends lésant leurs intérêts. Le conflit au départ transnational peut revêtir le caractère interétatique et dégénérer en conflit international. Les cas de la nationalisation du canal de Suez et les bombardements du Venezuela en 1902 en témoignent.

L'attraction des investissements est l'une des priorités de la mise en œuvre des législations tendant à améliorer le climat des affaires. La RDC ne fait pas exception. Il ne suffit pas de prévoir des mesures incitatives et attractives des investissements, mais encore, il faut en assurer la protection. Celle-ci peut revêtir plusieurs formes. La protection judiciaire seule est au centre du présent propos.

Cette étude serait orientée autrement si elle s'intéressait à un système judiciaire dans lequel les justiciables ont pleinement confiance aux institutions chargées de rendre justice. Tel n'est malheureusement pas encore le cas de la République démocratique du Congo. Estce une raison de décourager les éventuels investisseurs intéressés par le potentiel économique congolais en termes de domaines exploitables, de ressources naturelles, de la main d'œuvre et surtout d'un marché intérieur à plus de 70 millions d'habitants associé à une démographie galopante? Et les hommes d'affaires qui s'y trouvent déjà doivent-ils cesser toute activité au regard du tableau sombre du système judiciaire congolais? Loin de là.

Des mécanismes palliatifs de protection des investissements étrangers existent bel et bien en RDC. Si l'article 38 de la loi n004-2002 du 21 février 2002 portant code des investissements dispose que « tout différend entre un investisseur et la République démocratique du Congo relatif à un accord d'investissement ou à une autorisation d'investissement se règle préalablement à l'amiable. Si les parties ne parviennent pas à un règlement à l'amiable de leur différend dans un délai de 3 mois à compter de la première notification écrite demandant l'engagement de telles négociations, le différend sera réglé, à la requête de la partie lésée, conformément à une procédure d'arbitrage découlant :

- de la Convention du 18 mars 1965 pour le règlement des différends relatifs aux investissements entre Etats et Ressortissants d'autres Etats, (Convention CIRDI), ratifiée par la République Démocratique du Congo le 29 avril 1970 ou 
- des dispositions des Règlements du Mécanisme supplémentaire, si l'investisseur ne remplit pas les conditions de nationalité stipulées à l'article 25 de la convention CIRDI;

- du Règlement d'arbitrage de la Chambre de Commerce Internationale de Paris. ».

L'adhésion de la RDC à l'Ohada offre deux alternatives. La première est pourvue par l'existence d'une juridiction de cassation en dehors de la RDC qu'est la Cour Commune de Justice et d'Arbitrage. Le recours à cette juridiction communautaire permet de corriger les décisions judiciaires rendues par les tribunaux congolais qui seraient empreintes de partialité ou de subjectivité.

La CCJA dispose d'un mécanisme institutionnel en vue de la conciliation et de l'arbitrage. D'ailleurs, dans son rôle de tribunal arbitral, la CCJA s'appuie, comme les autres centres d'arbitrage de l'espace Ohada sur l'Acte uniforme du 11 mars 1999 relatif au droit de l'arbitrage.

Il faut relever ensuite que dans le cadre interétatique, la Cour permanente de Justice internationale ainsi que la Cour internationale de Justice ont eu à statuer sur des différends mettant en jeu la protection des investissements. La CPJI a examiné l'affaire Oscar Chinn mettant aux prises le Royaume-Uni, exerçant la protection diplomatique envers son national, Oscar Chinn, à la Belgique, puissance coloniale, sur des faits qui se sont déroulés sur le territoire congolais.

Tout récemment, la CIJ a tranché l'affaire Ahmadou Sadou Diallo. Dans cette cause, la Guinée a intenté une action judiciaire contre la RDC pour violation des droits sur la personne de Monsieur Diallo et sur ses deux entreprises Africom-Zaïre et Africonteners-Zaïre.

Analyser la protection internationale des investissements en RDC à l'aune de l'affaire Patrick Mitchell ${ }^{12}$ permet de jeter une lumière particulière sur une institution qui joue un rôle considérable dans le développement du droit international économique. Cette matière connait un faible fleurissement devant la Cour internationale de justice ${ }^{13}$. Le droit international de l'environnement, à la traine pendant un temps, bénéficie de nos jours d'un regain devant le principal organe judiciaire des Nations Unies ${ }^{14}$.

12 Il importe de rappeler qu'à la différence de certains litiges internationaux relatifs aux investissements tranchés par le CIRDI, l'affaire Patrick Mitchell se démarque singulièrement par sa procédure fort enrichissante. La différence précitée la place dans le cercle très fermé des affaires ayant été portées devant un Comité ad hoc après une première sentence arbitrale.

13 En dehors de la célèbre affaire de la Barcelona Traction, Power and Lights Limited mettant davantage en œuvre la protection diplomatique des actionnaires, on retiendra particulièrement l'affaire Elettronica sicula et, dans une certaine mesure l'affaire Ahmadou Sadou Diallo. lire CIJ, arrêt du 5 fevrier 1964 relatif à l'affaire Barcelona Traction, Power and Light limited, Rec. 1964; arrêt du 20 juillet 1989 en l'affaire de l'Elettronica sicula S.P.A(ELSI), Rec.1989; les arrêts du 24 mai 2007, du 30 novembre 2010 et du 12 juin 2012 relatifs à l'affaire Ahmadou Sadou Diallo, Rec.2007, Rec. 2010 et Rec.2012.

14 A l'inverse du droit international économique, il existe plusieurs décisions posant les principes du droit international de l'environnement. En outre des affaires du Droit de Corfou, de Certaines terres à phosphate à Nauru dans lesquelles la CIJ a énoncé des dictum relatifs à l'environnement, on retiendra l'affaire du Projet Gabcikovo-Nagymaros, l'affaire des Essais nucléaires, l'affaire de la licéité de l'emploi de l'arme nucléaire par un Etat dans un conflit armé, l'affaire de l'Usine de 
Fécond d'une jurisprudence abondante dans le domaine du droit international des investissements, le CIRDI est néanmoins méconnu du grand public. Bizarrement les juristes congolais n'échappent pas à l'ignorance de cette institution mettant en œuvre le principe de règlement pacifique des différends. Il est certes vrai que le CIRDI ne tranche pas les litiges interétatiques mais ceux mettant aux prises un Etat partie à la convention de Washington du 18 mars 1965 aux ressortissants d'un autre État partie. Mais il ne faut pas perdre de vue qu'en s'acquittant de sa mission, le CIRDI permet d'apaiser les tensions entre les États.

Si les États africains ne semblent pas s'intéresser des violations du droit international dont peuvent être victimes leurs ressortissants à l'étranger, peut être parce que les africains se targuent d'une faible participation aux investissements transnationaux, tel n'est pas le cas pour les autres nations.

La question de la protection des investissements des nationaux à l'étranger est à l'origine des ingérences multiformes et surtout la cause des conflits armées. Faut-il encore rappeler les blocus maritimes ainsi que les bombardements de ses portes qu'a été victime le Venezuela en 1902 de la part de l'Italie, l'Allemagne et du Royaume-Uni ${ }^{15}$. Le conflit de Suez rappelé ci-dessus est une parfaite illustration.

Quel intérêt y aura-t-il à analyser l'affaire Patrick Mitchell?

Théoriquement, elle constitue un cas illustratif du fonctionnement du CIRDI dans la mesure où elle met en œuvre les principes cardinaux de la justice arbitrale en soulignant les particularités du système CIRDI.

Il est proclamé qu'une sentence arbitrale est exécutoire et échappe à la procédure d'appel, le système CIRDI a introduit un mécanisme qui s'apparente à l'appel. En quoi consistet-il? Il s'agit de la procédure d'annulation de la sentence incriminée par un Comité ad hoc, un mécanisme par lequel on procède au réexamen de l'affaire ayant déjà fait l'objet de la sentence arbitrale dans des conditions précisées à l'article 52 de la Convention de Washington, sans faire intervenir les juridictions judiciaires.

L'intérêt de cette affaire réside également dans le fait qu'elle met aux prises la République démocratique du Congo à des particuliers étrangers. Elle utilise l'effectivité de la protection que les parties peuvent bénéficier devant cette institution sans recourir à l'aspect classique de règlement pacifique des différends.

L'affaire Patrick Mitchell revêt une importance particulière qui en justifie l'étude dans la mesure où elle met en exergue la difficile cohabitation entre différentes notions de droit international des investissements, particulièrement l'ambiguïté de la définition de l'investissement dans ce domaine. La particularité ne se limite pas là. Elle touche la procédure. Il est

pâte à papier sur le fleuve Uruguay, l'affaire de l'Epandage aérienne des herbicides, l'affaire de la Chasse à la baleine dans l'Antarctique et l'affaire de la Construction d'une autoroute sur le long du fleuve San Juan.

15 Ces bombardements sont à l'origine de l'interdiction du recours à la force pour recouvrement des dettes contractuelles comme dues à ses nationaux. Cette norme a été consacrée par la convention de La Haye de 1907 dite convention Drago-Porter. Lire Nguyen Quoc Dihn, Patrick Daillier, Mathias Forteau, Alain Pellet, Droit international public, $8^{\mathrm{èm}}$ édition, Paris, 2009, pp.1032-1033. 
vain de rappeler que les sentences arbitrales sont rendues en premier et dernier ressort. Il est bien entendu qu'il n'existe pas des voies de recours ordinaires ouvertes contre une sentence arbitrale. L'exception bien connue du recours en interprétation et en rectification d'erreurs matérielles applicables aux décisions judiciaires trouve application dans le cas d'espèce. Il y est étalé la protection qu'investisseurs et la RDC peuvent bénéficier de la part du CIRDI.

Il est intéressant de suivre les derniers développements de l'affaire Bernard Tapie ou mieux celle du Crédit lyonnais dans laquelle les arbitres sont poursuivis pour escroquerie en bande organisée pour avoir tranché un litige en octroyant à Bernard Tapie quatre cent millions d'euros au détriment de l'Etat français.

En l'espèce, la singularité de l'affaire Mitchell est de mettre en œuvre la procédure d'annulation d'une sentence arbitrale. Il ne s'agit pas d'un appel mais d'une véritable décision de reformulation de la sentence rendue par le tribunal arbitral.

D'un bon ton, il sera présenté les faits de la cause qui a opposé Monsieur Patrick Mitchell à la République démocratique du Congo, la procédure de ladite affaire avant de s'attarder sur le fond du litige.

\section{Faits et procédure}

L'affaire Patrick Mitchell intervient à un moment particulier de l'histoire de la République démocratique du Congo. Sans entrer dans le contexte politique d'alors, il faut rappeler que les faits surviennent à l'heure de la domination sans partage de la fameuse et inconstitutionnelle Cour d'ordre militaire.

Renversés par l'Alliance Démocratique pour la Libération du Congo du président Laurent-Désiré Kabila, le président Mobutu et son architecture institutionnelle et organique sont mis à terre ${ }^{16}$. Le nouvel homme fort de Kinshasa instaure un régime constitutionnel particulier avec le décret-loi constitutionnel 003 du 27 mai 1997 relatif à l'organisation et à l'exercice du pouvoir en République démocratique du Congo.

Des institutions nouvelles font leur apparition, notamment la fameuse Cour d'ordre militaire réputée pour ses décisions expéditives non susceptibles de recours. Cette juridiction s'est également rendue célèbre par le nombre élevé des causes aboutissant au prononcé et à l'exécution de la peine de mort ${ }^{17}$.

Trouvant racine à un moment particulier de l'histoire de la République démocratique du Congo, l'affaire Patrick Mitchell apparaît à la suite d'une décision de la Cour d'ordre militaire exécutée le 5 mars 1999. La relation entre le cabinet d'avocats Patrick Mitchell et as-

16 Sur la chute du maréchal Mobutu, on consultera utilement Martens Ludo, Kabila et la révolution congolaise. Panafricanisme ou néocolonialisme?, Tome 1, Anvers, 2002, particulièrement les pages 169 à 228 .

17 Plusieurs années après la cessation des activités de la Cour d'ordre militaire ainsi que l'application du moratoire en ce qui concerne l'exécution de la peine de la mort en République démocratique du Congo, le professeur André Mbata Betukumesu, alors député national élu, a tenté vainement de faire abolir la peine capitale en RDC. Le parlement ne l'a pas suivi. 
sociés et l'un de ses clients supposée illégale est à l'origine de la décision précitée de la Cour d'ordre militaire. La juridiction militaire soupçonnait le cabinet de garder des documents très compromettants contre la sécurité nationale congolaise. Ainsi, le 5 mars 1999, sur décision de cette juridiction, il a été procédé à la saisie des documents dudit cabinet, à la pose des scellés, et à l'arrestation des deux avocats, Me Risasi et Me Djunga. Les employés dudit cabinet ont été sommés de quitter leurs bureaux. Les avocats précités seront détenus pendant tout le long de l'instruction de l'affaire devant la Cour d'ordre militaire et ne seront remis en liberté que le 12 novembre $1999^{18}$.

C'est en cette date que la Cour d'ordre militaire rendra sa décision. Elle rejette les charges qui pesaient sur le cabinet Mitchell, restitue les biens qui avaient été saisis. Cependant, Monsieur Patrick Mitchell a considéré qu'il avait été victime d'une expropriation sans indemnisation de la part des autorités congolaises en violation de l'accord de promotion et de protection des investissements conclus en 1984 entre la République démocratique du Congo et les Etats-Unis d'Amérique. Il réclame réparation pour préjudices subis, incluant la perte des clients.

Du point de vue de la procédure, on retiendra que cette cause a été introduite le 6 octobre 1999. Dans sa requête introductive d'instance, le requérant sollicite du tribunal CIRDI de:

a) Déclarer que la République démocratique du Congo a, en violation du traité d'encouragement et de protection des investissements entre les Etats-Unis d'Amérique et le Congo, du droit congolais, et du droit international, exproprié les biens de Monsieur Patrick Mitchell;

b) Exiger de la RDC de dédommager Monsieur Patrick Mitchell pour tous les préjudices subis comme résultant de l'action de la République démocratique du Congo;

c) Allouer à Monsieur Patrick Mitchell tous les frais qu'il a encourus pour entreprendre l'arbitrage, incluant les honoraires des arbitres, les honoraires de chaque expert, les frais d'instance et les frais administratifs. ${ }^{19}$

Fondant expressément son action sur le traité d'encouragement et de protection des investissements, le tribunal CIRDI a procédé à l'examen de la cause en recourant à la désignation des arbitres. A ce sujet, il faut retenir que l'un des arbitres désigné en la personne de Monsieur Willard Z. Estey est décédé en cours d'instance le 25 janvier 2002 et a été remplacé par Monsieur Marc Lalonde le 13 février 2002. La composition ainsi que les parties se sont efforcés de déterminer la langue de la procédure. Le choix a été porté sur le français et l'anglais avec obligation pour la partie qui utilise l'une ou l'autre langue de produire une traduction, à l'exception des annexes.

Comme dit plus haut, l'affaire Patrick Mitchell est vallonnée d'éléments procéduraux intéressants et instructifs, puisqu'après la phase initiale qui a vu le tribunal arbitral prononcer une sentence arbitrale le 9 février 2004, la cause ne s'est pas clôturée là. La République

18 Patrick Mitchell c. République démocratique du Congo (2004), affaire nºARB/99/7 (CIRDI.).

19 Affaire nARB/99/7 (CIRDI), idem. 
démocratique du Congo a fait usage de toutes les possibilités que lui offrent la Convention de Washington ainsi que les règles de procédure devant le CIRDI pour solliciter d'abord la suspension de l'exécution de la décision du 9 février 2004. Celle-ci a été suspendue par la décision du $1^{\mathrm{er}}$ novembre 2004. Cette suspension a permis l'examen en toute sérénité de la cause par le Comité ad hoc. La persévérance et la ténacité de la RDC ont été récompensées puisque le Comité ad hoc a, par la décision du $1^{\mathrm{er}}$ novembre 2006, annulé la sentence arbitrale rendue en défaveur de la RDC.

\section{Le fond}

Affaire riche en rebondissement, l'examen du fond du cas Patrick Mitchell se fera en fonction des phases qu'a connu cette affaire. Un premier point reviendra sur les éléments déterminants de la première phase, essentiellement la notion de l'investissement et incidemment la compétence matérielle du tribunal CIRDI. Un deuxième point va s'intéresser de la phase de suspension de la sentence arbitrale, particulièrement les raisons de la suspension. Un troisième point s'appesantira sur la décision de l'annulation de la sentence Patrick Mitchell, notamment les raisons de l'annulation ainsi que leur incidence sur la notion de définition de l'investissement et de l'autorité des sentences arbitrales des tribunaux CIRDI qui sont censées rendues en premier et dernier ressort. Ce système a l'avantage d'assurer une protection égale aux deux parties litigantes.

\section{La sentence arbitrale et la décision de suspension}

L'affaire Patrick Mitchell a connu sa première décision le 9 février 2004 lorsque par une sentence arbitrale la République démocratique du Congo a été condamnée à payer au demandeur la somme en dollars américains de 750.000. Comme précisé ci-dessus, la sentence avait été suspendue par une décision du $1^{\text {er }}$ novembre 2004. Il est bon de s'attarder sur les raisons qui ont poussé les arbitres à condamner la RDC alors qu'objectivement parlant il y avait incompétence matérielle manifeste. Subsidiairement, on tentera de savoir plus sur les raisons de la suspension de la sentence précitée.

Attraite devant le tribunal CIRDI, la République démocratique du Congo ne s'est pas laissé faire. Elle a soutenu l'incompétence matérielle du tribunal CIRDI au motif que le service d'un avocat conseil ne rentre pas dans la catégorie de l'investissement. Par voie de conséquence, le tribunal CIRDI ne pourrait donc pas juger de cette affaire. La RDC est allée loin jusqu'à postuler des dommages et intérêts pour action téméraire et vexatoire de la part du demandeur Patrick Mitchell. Concernant la définition de l'investissement, le tribunal arbitral débute par affirmer qu'il n'avait pas de preuve suffisante de l'investissement ef- 
fectué par Patrick Mitchell en République démocratique du Congo ${ }^{20}$ avant d'aborder plus loin la question de l'existence de l'investissement.

Bien évidemment, il importe de relever que dans sa défense, la République démocratique du Congo a soulevé l'exception d'incompétence matérielle du tribunal CIRDI au motif que le litige soumis au tribunal CIRDI n'est pas relatif à l'investissement, conformément à l'article 25 de la Convention de Washington ${ }^{21}$. L'objection congolaise concerne « solely one to the qualification of Claimant's activity in the Congo as an investment"22.

Se défendant énergiquement, la RDC a soulevé un autre préalable de procédure lié au défaut d'épuisement des voies de recours internes ${ }^{23}$. Il n'a pas été opposé à la RDC le moyen de l'inexistence des recours judiciaires internes efficaces ou disponibles, comme dans l'affaire Diallo ${ }^{24}$. Le défendeur congolais s'est trompé d'enceinte. Devant le CIRDI, plusieurs mécanismes permettent au particulier dont l'investissement a souffert d'une violation de saisir directement cet organe sans passer par la protection diplomatique ni par l'épuisement des voies de recours internes.

Concrètement, le tribunal CIRDI a opposé à la RDC les termes de ses engagements avec les Etats-Unis d'Amérique. Le traité bilatéral ${ }^{25}$ sur l'encouragement réciproque et la protection des investissements impose, dans des termes permissif et facultatif, la condition de l'épuisement préalable des voies de recours internes. C'est donc un droit auquel le bénéficiaire peut renoncer d'exercer et non une obligation ${ }^{26}$.

20 Patrick Mitchell c. République démocratique du Congo (2004), Affaire nARB/99/7 (CIRDI) par. 17. Explicitement, les arbitres déclarent «Upon receipt and examination of all these written pleadings, the Tribunal came to the view that it had not received sufficient evidence concerning the investments made by the Claimant in the DRC.".

21 Ibid, par. 12. On peut lire « Mainly: that the ICSID is materially unqualified to know about a litigation which does not relate to "an investment".

22 Ibid, par. 17.

23 Dans l'affaire Interhandel, la CIJ a considéré que «[1]a règle selon laquelle les recours internes doivent être épuisés avant qu'une procédure internationale puisse être engagée est une règle bien établie du droit international coutumier; elle a été généralement observée dans les cas où un Etat prend fait et cause pour son ressortissant dont les droits auraient été lésés dans un autre Etat en violation du droit international. Avant de recourir à la juridiction internationale, il a été considéré en pareil cas nécessaire que 1'Etat où la lésion a été commise puisse y remédier par ses propres moyens, dans le cadre de son ordre juridique interne.», CIJ, Rec. 1959, p. 27.

24 CIJ, arrêt du 24 mai 2007 dans l'affaire Ahmadou Sadio Diallo, Exceptions préliminaires, Rec. 2007, par. 48 .

25 "It is further argued by Respondent that under Article III(3) of the BIT, Claimant was required to submit his request first to the competent judicial or administrative authority in the DRC.", Patrick Mitchell c. République démocratique du Congo, op. cit., par.33.

26 De l'aveu du tribunal CIRDI "this is manifestly not what results from the provision referred to. Under that rule, indeed, the recourse to state authorities is a right, but not an obligation of the investor, who is free to have direct recourse to the mechanism provided for in Article VII of the BIT for the settlement of disputes. Patrick Mitchell c. République démocratique du Congo, op. cit., par. 33. 
Le tribunal CIRDI s'est longuement attardé sur la question de l'investissement ${ }^{27}$ en rejetant la définition proposée par la RDC basée sur le droit interne congolais. Le tribunal a donc défini l'investissement suivant le TIB américano-congolais. Selon l'article 1 dudit accord:

"(c) 'investment' means every kind of investment, owned or controlled directly or indirectly, including equity, debt, and service and investment contracts; and includes:

(i) tangible and intangible property, including all property rights, such as liens, mortgages pledges, and real security;

(ii) a company or shares of stock or other interests in a company or interests in the assets thereof;

(iii) a claim to money or a claim to performance having economic value, and associated with an investment;

(iv) intellectual and industrial property rights, including rights with respect to copyrights, patents, trademarks, trade names, industrial designs, trade secrets and knowhow, and goodwill;

(v) licenses and permits issued pursuant to law, including those issued for manufacture and sale of products;

(vi) any right conferred by law or contract, including rights to search for or utilize natural resources, and rights to manufacture, use and sell products; and

(vii) returns which are reinvested.

Any alteration of the form in which assets are invested or reinvested shall not affect their character as investment. ${ }^{128}$

Soulignant la particularité de cette définition, le tribunal précise que "One striking feature of this definition is the fact that all items listed in this provision are mentioned for illustrative purposes. They do not have the effect of restricting in any manner the notion of an investment and the scope of the subject matter of the BIT." 29 La prémisse extensive posée par le tribunal laisse présager une conclusion incluant le service d'un avocat comme rentrant dans la catégorie de l'investissement. La démarche du tribunal ne s'arrête pas. Elle inclut les activités associées ou connexes. Selon les propres termes de la sentence sous examen « In addition to Article I, the BIT contains in Article II, dealing with "treatment of investment", a list of "associated activities", that are to be treated like investments and include, inter alia, "the making, performance and enforcement of contracts" (paragraph 2, letter c) ${ }^{30}$.

Poursuivant son raisonnement, le tribunal affirme que "With regard to the notion of "service contracts" as contained in the introductory part of Article I(c) of the BIT, the Tribunal notes that this notion is not further defined in the Treaty and that the exceptions listed

27 Ibid, particulièrement les paragraphes 45 à 48 .

28 Ibid, par. 45.

29 Ibid, par. 46.

30 Patrick Mitchell c. République démocratique du Congo, op. cit., par.46. 
in the Annex on behalf of the Republic of Zaïre are not helpful in this respect. Similarly, the associated activities referred to in Article II, which include "the making, performance and enforcement of contracts", do not contain any element of restriction in respect of service contracts" 31 .

La conclusion du tribunal est sans equivoque "the services typically offered by a firm providing legal advice as did the Claimant's firm are covered by the notion of services used by the BIT"32. Cette conclusion du tribunal arbitral n'a pas résisté au rouleau compresseur du comité ad hoc.

\section{La décision du comité ad hoc}

Rendue en premier et dernier ressort, selon la formule consacrée, la sentence arbitrale ne peut faire l'objet d'une annulation que dans des cas exhaustivement énumérés à l'article 52 de la Convention de Washington du 18 mars 1965. Selon le point 1 de la disposition précitée « chacune des parties peut demander, par écrit, au Secrétaire général l'annulation de la sentence arbitrale pour l'un quelconque des motifs suivants :

a) Vice dans la constitution du Tribunal;

b) Excès de pouvoir manifeste du Tribunal;

c) Corruption d'un membre du Tribunal;

d) Inobservation grave d'une règle fondamentale de procédure :

e) Défaut de motifs.

Obligatoire donc à l'égard des parties, la sentence arbitrale ne fait pas l'objet d'appel ni de voies de recours à l'exception de ceux prévus à l'article 52 susmentionné. La République démocratique du Congo, n'étant pas satisfaite de la sentence rendue le 9 février 2004, a introduit, en date du 7 juin 2004, une requête en suspension ${ }^{33}$ de l'exécution de ladite sentence ${ }^{34}$, de même qu'elle a initié une procédure en annulation de la sentence rendue en l'affaire Patrick Mitchell. Le Comité ad hoc a décidé en ces termes:

"Enforcement of the Award rendered on February 9, 2004 shall continue to be stayed according to Rule 54(2) of the Arbitration Rules, until the ad hoc Committee

31 Ibid, par. 52.

32 Ibid, par. 53.

33 En vertu du point 5 de l'article 52 « le Comité peut, s'il estime que les circonstances l'exigent, décider de suspendre l'exécution de la sentence jusqu'à ce qu'il se soit prononcé sur la demande en annulation. $\mathrm{Si}$, dans sa demande, la partie en cause requiert qu'il soit sursis à l'exécution de la sentence, l'exécution est provisoirement suspendue jusqu'à ce que le Comité ait statué sur ladite requête ».

34 Le Comité ad hoc a fait droit à la requête de la République démocratique du Congo en ordonnant la suspension de la sentence arbitrale du 9 février 2004, par la décision du 30 novembre 2004. Voir Patrick Mitchell c. République démocratique du Congo (2004), Affaire nARB/99/7 (CIRDI), Décision sur la suspension de l'exécution de la sentence. 
issues its decision on the application for annulment filed by the Democratic Republic of Congo"35

D'orès et déjà, il importe de souligner que l'appel n'étant pas de mise devant les tribunaux CIRDI, il revient au Comité ad hoc de jouer une partie d'équilibriste pour ne pas faire l'œuvre du juge d'appel. La mission particulière qui lui est dévolue n'échappe pas à la conscience du Comité ad hoc. Celui-ci s'est empressé de le rappeler « No one has the slightest doubt - all the ad hoc Committees have so stated, and all authors specializing in the ICSID arbitration system agree - that an annulment proceeding is different from an appeal procedure and that it does not entail the carrying out of a substantive review of an award." 36 Le Comité ad hoc poursuit en soulignant la particularité de l'affaire Patrick Mitchell « the grounds invoked by the Respondent in respect of the Award are sometimes based on arguments which touch upon the merits of the case. The ad hoc Committee is fully aware of the risk to which the nature of this case exposes it." 37

Précisant sa mission, le Comité ad hoc martèle "Nor is there any doubt that the grounds for annulment set out in Article 52 must be examined in a neutral and reasonable manner, that is, neither narrowly nor extensively. An ad hoc Committee should not decide to annul an award unless it is convinced that there has been a substantial violation of a rule protected by Article 52." 38

Faisant asseoir initialement sa requête d'annulation de la sentence arbitrale du 9 février 2004 sur cinq (5) bases $^{39}$, la République démocratique du Congo va, au cours de la procédure d'annulation, abandonner deux pour maintenir trois (3). Il s'agit d'un « excès de pouvoir manifeste quant à la compétence du Tribunal arbitral au regard de la définition de l'investissement; excès de pouvoir manifeste quant au droit applicable au différend; défaut de

35 Voir Patrick Mitchell c. République démocratique du Congo (2004), Affaire nARB/99/7 (CIRDI), Décision d'annulation du $1^{\mathrm{er}}$ novembre 2006, par. 9.

Ibid, par. 19.

37 Ibid.

38 Ibid.

39 "(i) Manifest excess of powers with regard to the Arbitral Tribunal's jurisdiction in respect of the definition of investment;

(ii) Manifest excess of powers with regard to the identity of the Claimant and with regard to the "arising directly out of" relationship between the dispute and the investment;

(iii) Manifest excess of powers with regard to the law applicable to the dispute;

(iv) Manifest excess of powers with regard to the failure to apply Article VII(3) of the BIT to the dispute for purposes of determining the admissibility of the request of Mr. Patrick Mitchell; and

(v) Failure to state reasons for the jurisdiction ratione materiae of the Arbitral Tribunal with regard to the identity of the Claimant, the characterization of the measures under dispute as expropriation, and the amount of compensable damages." Ibid, par. 15. 
motifs quant à la compétence ratione materiae du Tribunal arbitral, quant à la qualification d'expropriation des mesures litigieuses et quant au montant du dommage réparable. ${ }^{40}$

S'opposant énergiquement à l'action de la RDC, le demandeur originel soutient que le recours en annulation introduit par la RDC a un caractère abusif et qu'en réalité le Congo recherche un contrôle du fond de la sentence arbitrale ${ }^{41}$.

Abordant l'examen de l'excès de pouvoir manifeste et de défaut de motifs quant à la compétence du tribunal CIRDI en raison du fait qu'il y aurait pas d'investissement dans l'affaire en cause, le Comité ad hoc a recouru aux critères Salini ${ }^{42}$ en s'appuyant particulièrement sur la contribution au développement économique de l'Etat hôte. Le Comité ad hoc commence son analyse de manière fracassante et précise le nœud du litige « Indeed, in the case at hand the question is not so much one of determining whether there is an "arising directly out of" relationship, but rather whether there is an "investment."43

Prenant à contre-pied la sentence du 9 février 2004, le Comité ad hoc affirme qu'à son opinion « in view of the absence in the Convention of an explicit definition of the concept of investment, it is in the parties' agreement or in the applicable investment treaty that one should look for such definition, whether it is broad or less broad. In doing so, the fact that a State has not made use of the notification option provided for under Article 25(4) of the Convention may not be understood to mean that that State has taken a certain position regarding the very concept of investment. It is then necessary to verify the conformity of the concept of investment as set out in the parties' agreement or in the BIT with the concept of investment in the Washington Convention, as this latter results from the interpretation of the Convention in accordance with Article 31.1 of the Vienna Convention on the Law of Treaties, as well as from ICSID case law, to the extent the latter may contribute to defining the concept." 44

Dans son œuvre d'annulation, le Comité ad hoc est revenu sur les caractéristiques d'un investissement, après avoir présenté les définitions proposées par le traité bilatéral d'encouragement réciproque des investissements conclu entre la RDC et les Etats-Unis d'Amérique $^{45}$. Le Comité s'est appesanti sur le critère de contribution au développement économique de l'Etat hôte avec la précision que Patrick Mitchell n'a pas contribué au développe-

40 Patrick Mitchell c. République démocratique du Congo (2004), Affaire nARB/99/7 (CIRDI), Décision d'annulation du $1^{\mathrm{er}}$ novembre 2006, par. 17.

41 Ibid, par. 18. La partie Patrick Mitchell avance que « the Application for Annulment to be abusive, arguing that the Respondent seeks to review the merits of the Award. He therefore requests that the ad hoc Committee reject all grounds for annulment invoked by the DRC and, pursuant to Article 52(4) and of Chapter VI of the Convention, have the Respondent bear all the costs of this proceeding, including legal fees.".

42 Salini Construttori S.p.A. and Italstrade S.p. A. c. Morocco, affaire CIRDI n ARB/00/4, décision sur la compétence du 23 juillet 2001, para.52.

43 Patrick Mitchell c. République démocratique du Congo (2004), Affaire nARB/99/7 (CIRDI), Décision d'annulation du $1^{\mathrm{er}}$ novembre 2006, par. 25.

44 Ibid.

45 Voir Patrick Mitchell c. République démocratique du Congo (2004) ... loc. cit., par. 26. 
ment de la RDC. Alors que la sentence du 9 février 2004 souligne l'absence d'une déclaration particulière sur la définition de l'investissement au regard de la convention de Washington du 18 mars 1965, le Comité ad hoc soutient au regard de la condition de la contribution au développement de l'Etat que le demandeur « does not constitute one of the criteria for the existence of an investment $»^{46}$

Faisant effort de faire asseoir sa thèse, le Comité ad hoc s'est livré à une démonstration jurisprudentielle en citant des sentences CIRDI qui ont fait application du critère de la contribution au développement de l'Etat hôte. Le Comité s'est tour à tour reposé sur les affaires $^{47}$ Salini $^{48}$, Fedax $^{49}$ et $S C O B^{50}$. Quoique reconnaissant un certain élargissement de la notion de l'investissement dans les affaires précitées sans que cela n'altère la nature fondamentale de cette caractéristique, le Comité ad hoc poursuit son raisonnement. Il spécifie que « in another group of cases where the contribution to the economic development of the host State had not been mentioned expressly, it was doubtless covered by the very purpose of the contracts in question - all of which were State contracts - which had an obvious and unquestioned impact on the development of the host State". ${ }^{51}$ Ce qui revient déjà à souligner l'absence d'un contrat liant directement le demandeur Patrick Mitchell à la République démocratique du Congo. Ainsi donc, si dans les causes mettant directement en relief la relation contractuelle entre un particulier et un Etat donné, la caractéristique de la contribution au développement de l'Etat a été retenue, il en sera forcément ainsi dans une cause où ce lien est inexistant.

46 Patrick Mitchell c. République démocratique du Congo (2004), Affaire nºRB/99/7 (CIRDI), Décision d'annulation du $1^{\text {er }}$ novembre 2006, par. 27.

47 Ibid, par. 30.

48 Explicitement le Comité reprend un passage de la sentence Salini « in the Salini case, the contribution to the economic development of the host State was explicitly set as a "criterion" for an investment which was subsequently taken into account in respect of the construction of a highway, which led to the conclusion that the highway was clearly of public interest". Patrick Mitchell $c$. République démocratique du Congo (2004), Affaire n ARB/99/7 (CIRDI), Décision d'annulation du $1^{\mathrm{er}}$ novembre 2006, par. 30.

49 Selon la sentence Fedax N.V. c. Venezuela "It is quite apparent that the transactions involved in this case are not ordinary commercial transactions and indeed involve a fundamental public interest [...] There is clearly a significant relationship between the transaction and the development of the host State". C'est le Comité ad hoc qui souligne. Patrick Mitchell c. République démocratique $d u$ Congo (2004), Affaire n ${ }^{\circ} \mathrm{ARB} / 99 / 7$ (CIRDI), Décision d'annulation du $1^{\text {er }}$ novembre 2006, par. 30.

50 Dans la sentence Ceskoslovenska obchodni, a. s. [CSOB] c. la Slovaquie, il a été décidé que «Under certain circumstances a loan may contribute substantially to a State's economic development [...] [The] undertaking involved a significant contribution by CSOB to the economic development of the Slovak Republic within the meaning of the Convention." C'est le Comité ad hoc qui souligne. Patrick Mitchell c. République démocratique du Congo (2004), Affaire nº ARB/99/7 (CIRDI), Décision d'annulation du $1^{\mathrm{er}}$ novembre 2006, par. 30.

51 Ibid. 
Peaufinant sa motivation, le Comité ad hoc a fait recours à un grand doctrinaire pour galvaniser sa position. Il pourrait être opposé à la décision d'annulation de la sentence arbitrale dans l'affaire Patrick Mitchell de faire recours à une source non formelle du droit international. La contestation n'est pas dénuée de tout fondement dans la mesure où le CIRDI fait également application du droit international. La lecture du point 1 de l'article 42 pourrait laisser un triomphalisme émerger ${ }^{52}$. La Convention de Washington se réfère expressément aux principes de droit international que renferment les sources formelles énumérées de manière non exhaustive à l'article $38 \mathrm{du}$ Statut de la Cour internationale de Justice. Elle cite également au point 3 de l'article 42 précité la possibilité pour le Tribunal de statuer, si les parties y consentent, ex aequo et bono. Il est certes vrai qu'au regard de la particularité du droit des gens, un droit interétatique, il n'est pas possible qu'une frange d'auteurs en soit géniteur. Néanmoins les termes de l'article 38 du statut de la CIJ sont éloquents. Il est question d'un moyen auxiliaire de détermination de la règle de droit. Ainsi, après avoir démontré jurisprudence à l'appui la pertinence de sa motivation, le Comité ad hoc évoque donc la doctrine en soutien. Il cite Schreuer qui considère la contribution au développement économique de l'Etat hôte comme " the only possible indication of an objective meaning" of the term "investment.»" ${ }^{53}$

Sans se contenter de ce « secours » doctrinal, le Comité ad hoc assène à la sentence du 9 février 2004 un coup de massue en consacrant la suprématie de la Convention de Washington du 18 mars 1965 sur les conventions particulières quant à la définition d'un investissement. Ainsi, « the parties to an agreement and the States which conclude an investment treaty cannot open the jurisdiction of the Centre to any operation they might arbitrarily qualify as an investment. It is thus repeated that, before ICSID arbitral tribunals, the Washington Convention has supremacy over an agreement between the parties or a BIT $»^{54}$.

Percevant le danger que peut susciter le critère de la contribution au développement de l'Etat d'accueil dans la définition de l'investissement, le Comité ad hoc a précisé que « l'existence d'une contribution au développement économique de l'État d'accueil comme caractéristique essentielle - bien que non suffisante - ou critère incontestable de l'investissement, ne signifie pas que cette contribution doit toujours être importante ou fructueuse; et bien sûr les tribunaux CIRDI n'ont pas à évaluer la contribution réelle de l'opération en cause. Il suffit que l'opération contribue d'une manière ou d'une autre au développement

52 D'après le point 1 de l'article 42 de la convention de Washington du 18 mars 1965 « Le Tribunal statue sur le différend conformément aux règles de droit adoptées par les parties. Faute d'accord entre les parties, le Tribunal applique le droit de l'Etat contractant partie au différend - y compris les règles relatives aux conflits de lois - ainsi que les principes de droit international en la matière ». Le soulignement vient de l'auteur.

53 Christopher Schreuer, The ICSID Convention: a Commentary, 2001, p. 124, cite par le Comité ad hoc in. Patrick Mitchell c. République démocratique du Congo (2004), Affaire nºRB/99/7 (CIRDI), Décision d'annulation du $1^{\text {er }}$ novembre 2006, par. 30.

54 Ibid, par.31. 
économique de l'État d'accueil, ce concept de développement économique étant de toute façon très vaste mais aussi variable selon les cas. » ${ }^{55}$

Alors que la sentence du 9 février 2004 présente le service d'avocat comme une catégorie de service ${ }^{56}$ inclue dans le traité américano-congolais intégrant la définition de l'investissement, le Comité ad hoc démolit cette position. Il souligne que « The ad hoc Committee cannot but observe two things: first, that resolving the question of application of the concept of service within the meaning of the Treaty to legal consulting services by stating that this concept embraces "any service provided by a foreign investor," is to fall into a vicious circle, inasmuch as the Award does not explain why in this case the Claimant was an investor; and second, that item I(c) of the Treaty speaks of "every kind of investment, [...] including [...] service and investment contracts," and not of "every kind of service.» ${ }^{57}$

Le traité bilatéral entre les Etats-Unis et la République démocratique du Congo parle bien de toute sorte d'investissement, [...] incluant [...] les contrats et services relatifs à l'investissement et non de tout service, sans autre précision. La sentence du 9 février 2004 a donc emprunté un raccourci pour insérer le service juridique fourni par Patrick Mitchell à ses clients dans la catégorie de l'investissement, justifiant ainsi la compétence ratione materiae du tribunal.

Relevant que la sentence ne prouve pas le contenu du service du cabinet Patrick Mitchell et associés qui pourrait justifier que son activité soit qualifiée d'investissement ${ }^{58}$, le Comite ad hoc affirme « it would be necessary for the Award to indicate that, through his know-how, the Claimant had concretely assisted the DRC, for example by providing it with legal services in a regular manner or by specifically bringing investors." 59

Le risque de considérer un cabinet d'avocat comme un investissement est trop élevé. Le comité conclut donc à un défaut de motifs «dans le sens d'une insuffisance de motifs telle qu'elle affecte sérieusement la cohérence du raisonnement quant à l'existence d'un investissement selon l'article 25(1) de la Convention de Washington et le Traité bilatéral entre les

55 Ibid, par. 33. Selon le Comité ad hoc « the existence of a contribution to the economic development of the host State as an essential - although not sufficient - characteristic or unquestionable criterion of the investment, does not mean that this contribution must always be sizable or successful; and, of course, ICSID tribunals do not have to evaluate the real contribution of the operation in question. It suffices for the operation to contribute in one way or another to the economic development of the host State, and this concept of economic development is, in any event, extremely broad but also variable depending on the case".

56 "The Arbitral Tribunal considered that the concept of service must be broad in scope, including "any service provided by a foreign investor," and especially the services normally provided by a legal consulting firm, as was the case of the Claimant's firm". Patrick Mitchell c. République démocratique du Congo (2004), Affaire nARB/99/7 (CIRDI), Sentence du 9 février 2004, par. 53.

57 Patrick Mitchell c. République démocratique du Congo (2004), Affaire nºRB/99/7 (CIRDI), Décision d'annulation du Sentence du $1^{\mathrm{er}}$ novembre 2006 par. 37.

58 Ibid, par.38.

59 Ibid, par. 39. 
États-Unis d'Amérique et la République Démocratique du Congo, de laquelle dépendait la compétence du Tribunal ${ }^{60}$.

Abordant la question d'excès de pouvoir ou d'excès manifeste de pouvoir, le Comité relève des raccourcis très dangereux utilisés par la sentence du 9 février 2004, concernant notamment le fonds transféré aux Etats-Unis d'Amérique mais non réinvesti en République démocratique du Congo. «The finding of the Arbitral Tribunal on this point is rather surprising, in that Article I(c) of the BIT includes in the concept of investment under item (vii) "returns which are reinvested." Consequently, 'non-reinvested' returns cannot be included in the concept of investment and be protected by the Treaty.» ${ }^{61}$ Le Comité ad hoc fustige ainsi la mauvaise interprétation des traités qu'a faite la sentence arbitrale du 9 février $2004{ }^{62}$ Le Comité $a d$ hoc conclut à un excès de pouvoir de la part des arbitres qui avaient rendu la sentence du 9 février 2004. Il affirme donc «The ad hoc Committee is thus inclined to believe that the Arbitral Tribunal forced the concept of investment in the case at hand in order to affirm its jurisdiction" 63 , puis achève " The fact remains, however, that this combination of flaws in the Award is such that an excess of power on the part of the Arbitral Tribunal must be acknowledged." 64

Après une démonstration logique, la décision du Comité ad hoc tombe comme un couperet «

The ad hoc Committee is thus obligated to annul the Award of February 9, 2004 due to the failure to state reasons and manifest excess of powers committed by the Arbitral Tribunal in its decision to declare itself competent $»^{65}$. Ainsi donc en reconnaissant qu'il y a eu défaut de motivation et excès de pouvoir dans la sentence du 9 février 2004 par le fait que le Tribunal CIRDI s'était déclaré compétent, le Comité ad hoc estima en bon droit qu'il était superfétatoire de poursuivre l'analyse des autres moyens développés par la défende-

60 «The ad hoc Committee thus concludes that the Award is tainted by a failure to state reasons, in the sense that the inadequacy of reasons is such that it seriously affects the coherence of the reasoning as to the existence of an investment in accordance with Article 25(1) of the Convention and the Bilateral Treaty between the United States of America and the Democratic Republic of Congo, on which relied the jurisdiction of the Arbitral Tribunal", ibid, par. 41.

61 Patrick Mitchell c. République démocratique du Congo (2004), Affaire n ARB/99/7 (CIRDI), Décision d'annulation du Sentence du $1^{\text {er }}$ novembre 2006 par. 43.

62 Ibid. Selon le Comité ad hoc «This a contrario interpretation of one of the items on an enumerative list, even one that is not exhaustive, is fully in keeping with the logic and the spirit of a BIT and is equivalent to an interpretation "in good faith in accordance with the ordinary meaning to be given to the terms of the treaty in their context and in the light of its object and purpose," in accordance with Article 31(1) of the Vienna Convention on the Law of Treaties of 1969, which codifies a rule of customary international law ».

63 Patrick Mitchell c. République démocratique du Congo (2004), Affaire n ARB/99/7 (CIRDI), Décision d'annulation du Sentence du $1^{\text {er }}$ novembre 2006 par. 46.

64 Ibid.

65 Ibid, par. 48. 
resse, la République démocratique du Congo. Cependant, la décision du $1^{\text {er }}$ novembre 2006 a débattu des questions liées à l'expropriation ainsi qu'à l'indemnisation ${ }^{66}$.

Rendue le $1^{\text {er }}$ novembre 2006, la décision du Comité ad hoc a été encensée et critiquée par la doctrine. On ne manquera pas de stigmatiser que, du point de vue jurisprudentiel, la sentence produite dans l'affaire Pey Casada critiquant les critères Salini, particulièrement la question de la contribution au développement économique de l'Etat d'accueil de l'investissement, figure en bonne place des offensives contre la sentence Mitchell

La décision du Comité ad hoc a été saluée dans la jubilation par Florian Grisel. Ce dernier considère que ladite décision « ... semble fondée du point de vue juridique, et justifiée au plan économique et politique $» .{ }^{67}$ Un autre auteur signale que le tribunal a identifié au moins deux critères pour qu'un investissement contribue au développement économique du pays hôte : " l'investissement doit bénéficier à l'intérêt public, et il doit y avoir un transfert de savoir-faire $\gg{ }^{68}$ Les assauts les plus violents sont venus notamment de Julien et Dany Khayat $^{69}$ ainsi que d'Emmanuel Gaillard ${ }^{70}$.

Pour les premiers cités, le Comité ad hoc aurait fait une analyse peu satisfaisante aussi bien sur la forme que le fond ${ }^{71}$. «En rendant sa décision, le comité ne s'assure pas de la bonne application du droit par le tribunal mais décide la substance du droit à appliquer, substance qui peut contenir plusieurs critères pour retenir la notion d'investissement mais qui reste un choix du tribunal au fond et en aucun cas celui d'un comité ad hoc en matière d'annulation. La détermination du contenu du fond du droit illustre le fait que dès les prémices de son analyse, le comité se dirige vers une analyse des faits de l'affaire et non de la bonne application du droit» ${ }^{72}$. Ainsi, il apparaît que pour ces auteurs, le Comité a outrepassé ses prérogatives. En réalité, les deux auteurs précités se livrent à un acharnement contre l'œuvre du Comité ad hoc. Ils soutiennent que même en l'absence des fonds réinvestis en $\mathrm{RDC}$, le tribunal se serait déclaré compétent par d'autres éléments ${ }^{73}$. Comment le pourraitil dans la mesure où l'investissement n'existe pas et que ce dernier est la condition sine qua

66 Ibdi, par. 49-65.

67 Florian Grisel, « Arbitrage d'investissement et promotion du développement économique: une étude de cas », dans OECD Global Forum on International Investment VII, 27-28 March 2008, p. 1.

68 Garcia-Bolivar Omar E., « Définir un investissement au titre du CIRDI: pourquoi le développement économique devrait être l'élément essentiel », dans Investment Treaty News, n³, vol.2, avril 2012, p. 4.

69 Julien Fouret et Dany Khayat, « CIRDI », dans Revue Québécoise de Droit international, 2006, pp. 271-347.

70 Emmanuel Gaillard, « Centre International pour le Règlement des Différents relatifs aux Investissements, 2 chronique des sentences arbitrales », dans Chroniques, janvier-février-mars 2009, pp. $333-416$.

71 Julien Fouret et Dany Khayat, « CIRDI », dans Revue Québécoise de Droit international, 2006, p. 341.

72 Ibid, p.342.

73 Ibid,p. 345. 
posée par l'article 25 de la Convention de Washington du 18 mars 1965 pour déterminer la compétence ratione materiae du tribunal CIRDI? Ils s'autorisent une spéculation concernant les retours de fonds de Monsieur Patrick Mitchell qui ne sont pas réinvestis en RDC en affirmant qu'"ils auraient été utilisés dans le paiement des frais de fonctionnement du cabinet" ${ }^{\text {"74. }}$.

Se faisant, Fouret et Khayat ajoutent un élément qui n'existe pas dans la sentence arbitrale. Cette dernière a considéré les retours de fonds aux Etats-Unis comme constitutifs de l'investissement sans chercher à savoir s'ils étaient réinvestis ou pas, contrairement à ce que prévoit le TBI américano-congolais. Cet accord bilatéral les admet à titre d'investissement à la seule condition qu'ils soient réinvestis. Tel n'est pas le cas. De son côté Emmanuel Gaillard, conseil de la partie Patrick Mitchell dont les critiques contre la décision du $1^{\text {er }}$ novembre 2006 peuvent légitimement souffrir du subjectivisme, pourfend cette dernière et la considère comme « plus proche d'une révision ${ }^{7}{ }^{75}$

Dominique Carreau n'est pas non plus tendre avec la décision du $1^{\text {er }}$ novembre 2006, ni à l'égard de celles qui lui sont assimilées intégrant la contribution au développement de l'Etat hôte comme critère déterminant de la définition de l'investissement. Il soutient que « D'autres sentences CIRDI ont été inutilement plus exigeantes [...] en requérant que l'opération en cause « contribue au développement économique du pays d'accueil » (V. par exemple : Sent. 11 juill. 1997, Fedax c/Venezuela; plus récemment : Décis. Comité ad hoc 1er nov. 2006, Patrick Mitchell, (...). On ne peut s'empêcher ici de noter l'inutilité de ce critère additionnel souvent avancé : soit il ne peut manquer d'apparaître comme superflu et tautologique (tout investissement n'apporte-t-il pas par définition un «plus » au pays hôte?) soit, de surcroît, il se révèle d'un maniement particulièrement subjectif (ainsi une sentence CIRDI plus récente du 31 juin 2007 refusa justement de le prendre en compte (M.CI Power Group L.C. and New Turbine c/Équateur); en tout état de cause, il se révèle un facteur d'insécurité juridique fâcheux, de sorte que, rien qu'à ce titre, il devrait être écarté » ${ }^{76}$.

Avec une telle affirmation, Dominique Carreau ne prouve pas spécifiquement en quoi le service d'un cabinet d'avocat, qui n'entre pas dans le cadre formel des investissements tracé par le TBI américano-congolais et sans avoir servi l'intérêt public, pourrait contribuer au développement économique de la RDC. Plus modérée est la position d'Andrew Newcombe et Lluis Paradell "The ad hoc annulment committee in Mr. Patrick Mitchell v. Congo (Decision on the Application for the Annulment of the Award, 1 Nov. 2006) followed Salini and stated that the "existence of a contribution to the economic development of the host State as

74 Ibid.

75 Emmanuel Gaillard, "Chronique des sentences arbitrales », dans Journal de droit international (clunet), $n^{\circ} 1,2007, \mathrm{p} .81$.

76 Dominique Carreau, « Investissements », dans Répertoire international Dalloz, août 2008, p.13. 
an essential - although not sufficient - characteristic' of an investment for the purposes of the ICSID Convention". 77

N'en déplaisent aux Julien Fouret, Dany Khayat, Dominique Carreau, la décision du Comité ad hoc assure l'équilibre des intérêts et permet ainsi de fermer la porte à une extension abusive de la notion d'investissement.

\section{CONCLUSION}

Le CIRDI joue un rôle majeur dans la circulation des biens et des services ainsi que dans les échanges commerciaux parmi les Etats. Il est incontestable que cette juridiction assure une sécurité judiciaire aux investissements entrepris sur la face de la planète. Les investisseurs se sentent encouragés à investir dans les Etats qui disposent des ressources naturelles, des débouchés, un marché intérieur important ainsi qu'une main d'œuvre facile et abondante. L'assurance de l'intervention d'une juridiction impartiale pour trancher les litiges naissant éventuellement sur le territoire de l'Etat d'accueil triomphe des réticences.

Sans être désemparés du fait que les juridictions locales ne seront pas en mesure de pourvoir à une protection adéquate des investissements, les milieux d'affaires trouvent dans le CIRDI un gage de stabilité, d'assurance et de sécurité. Ils ont la certitude qu'une hypothétique expropriation des biens investis serait équitablement indemnisée.

Alors que la notion de l'investissement pose problème et peut donc constituer une incertitude dans la protection des investissements, la décision du $1^{\mathrm{er}}$ novembre 2006 dans l'affaire Patrick Mitchell apporte une contribution majeure dans la confiance que les plaideurs, Etats et particuliers, peuvent avoir au CIRDI. Elle annonce et met à nu un système hypercomplexe, à la différence du système de droit interne ou de tout autre arbitrage classique dans lequel la sentence arbitrale ne peut faire l'objet de voies de recours ordinaire. Le système CIRDI permet ainsi d'annuler une sentence arbitrale lorsque les conditions posées à l'article 52 de la Convention de Washington du 18 mars 1965 sont réunies. Malheureusement, la contradiction des sentences est susceptible de fragiliser le système CIRDI, ce qui demeure un écueil.

\section{BIBLIOGRAPHIE}

\section{A. Textes officiels}

1. Texte international

Accord de promotion et de protection des investissements conclus en 1984 entre la République démocratique du Congo et les Etats-Unis d'Amérique;

77 Andrew Newcombe and Lluis Paradell, Law and Practice of Investment Treaties, Austin, Boston, Chicago, New York, The Netherlands, 2009, p. 67, note 420. 
La protection internationale des investissements en République démocratique du Congo à l'aune de l'affaire Patrick Mitchell devant le CIRDI

Convention de Washington du mars 1965 relative au Règlement des Différends Internationaux relatifs Investissements entre les États et ressortissants d'un autre État.

2. Jurisprudence

a) $\mathrm{CPJI}$

CPJI, Affaire du Lotus, arrêt du 7 septembre 1927, série A, $n^{\circ} 10$;

CPJI, avis consultatif $\mathrm{n}^{\circ} 4$ du 7 février 1923 relatif aux Décrets tunisiens et marocains de nationalité, Série B.

\section{b) CIJ}

CIJ, Arrêt du 24 mai 2007 relatif à l'affaire Ahmadou Sadou Diallo (Guinée c/République démocratique du Congo), Rec. 2012;

CIJ, Arrêt du 30 novembre 2010 à l'affaire Ahmadou Sadou Diallo (Guinée c/République démocratique du Congo), Rec. 2010;

CIJ, Arrêt du 12 juin 2012 relatif à l'affaire Ahmadou Sadou Diallo, Rec.2007;

CIJ, Avis consultatif du 9 juillet 2004 dans l'affaire des Conséquences juridiques de l'édification d'un mur dans le territoire palestinien occupé, CIJ, Rec. 2004;

CIJ, arrêt du 20 juillet 1989 dans l'affaire de l'Electtronica Sicula S.p.a.(Etats-Unis d'Amérique c/ Italie), Rec. 1989.

CIJ, arrêt du 5 février 1970 dans l'affaire de la Barcelona Traction, Lights and Power Company, Limited (Nouvelle requête 1962),(Belgique c/ Espagne, 1962, Deuxième phase), Rec. 1970.

CIJ, arrêt du 6 avril 1955 relatif à l'affaire Nottebohm, Rec.1955.

\section{c) CIRDI}

CIRDI, Victor Pey Casado et Fondation « Presidente Allende » c. République du Chili, affaire CIRDI n -98/2, sentence du 22 avril 2008;

CIRDI, M.C.I. Power Group L. C. and New Turbine, Inc c. Equateur, affaire CIRDI n ${ }^{\circ} \mathrm{ARB} / 03 / 6$, sentence du 31 juillet 2007.

CIRDI, Malaysian Historical Salvors, SDN, BHD c. Malaisie, affaire CIRDI n ARB/05/10, sentence du 17 mai 2007;

CIRDI, Saipem S.p.A. c. La République populaire du Bangladesh, affaire CIRDI n ARB/05/07, décision sur la compétence et recommandation sur les mesures provisoires du 21 mars 2007;

CIRDI, Patrick Mitchell c. République démocratique du Congo (2004), Affaire nºARB/99/7 (CIRDI), Décision d'annulation du $1^{\mathrm{er}}$ novembre 2006;

CIRDI, Helnan International Hotels A/S c. République arabe d'Egypte, affaire CIRDI n ${ }^{\circ} \mathrm{ARB} / 05 / 19$, décision sur la compétence du 17 octobre 2006;

CIRDI, Jan de Nul N.V. and Dredging International N.V. c. République arabe d'Egypte, affaire CIRDI $\mathrm{n}^{\circ} \mathrm{ARB} / 04 / 13$, décision sur la compétence du 16 juin 2006; 
CIRDI, Joy Mining Machinery Limited c. République arabe d'Egypte, affaire CIRDI n ${ }^{\circ}$ ARB/03/11, sentence sur la compétence du 6 août 2004;

CIRDI, Salini Construttori S.p.A. and Italstrade S.p. A. c. Morocco, affaire CIRDI n ARB/00/4, décision sur la compétence du 23 juillet 2001;

CIRDI, Ceskoslovenska Obchodni Banka c. République slovaque, affaire CIRDI n97/4, décision sur la compétence, 24 mai 1999;

CIRDI, Fedax c. Venezuela, Affaire nARB/96/3, décision sur la compétence du 11 juillet 1997;

CIRDI, MINE v. Guinea, décision du 6 janvier 1988;

Swiss Aluminium v. Iceland, decision du 6 mars 1985;

CIRDI, Kaiser Bauxite v. Jamaica, Décision du 6 juillet 1975; RSM Production Corporation c. République centrafricaine, $\mathrm{ARB} / 07 / 2$;

CIRDI, Affaires Holiday Inns v. Morocco, décision du 12 mai 1974, ICSID Report 650;

d) Commission africaine des droits de l'homme et des peuples

Communication 221/98, Afred B. Cudjoe c/Ghana;

Communication 135/94, Kenya Human Rights Commission c.Kenya;

Communication 90/93, Paul S. Haye c/Gambie;

Communication 60/91, Constitutional Rights Project c/Nigeria;

Communication 18/88, El Hady Boubacar Diawara c Benin;

\section{B. Ouvrages, Articles et autres documents}

Dominique Carreau, « Investissements », dans Répertoire international Dalloz, août 2008.

Helène Fouillet, « Le consentement de l'Etat à l'Arbitrage du Centre International Règlement des Différends relatifs aux Investissements».

Julien Fouret et Dany Khayat, « CIRDI », dans Revue Québécoise de Droit international, 2006.

Emmanuel Gaillard, «Centre International pour le Règlement des Différents relatifs aux Investissements, 2 chronique des sentences arbitrales ", dans Chroniques, janvier-février-mars 2009, pp. 333-416.

Emmanuel Gaillard, " Chronique des sentences arbitrales », dans Journal de droit international (clunet), $n^{\circ} 1,2007$.

Garcia-Bolivar Omar E., « Définir un investissement au titre du CIRDI: pourquoi le développement économique devrait être l'élément essentiel », dans Investment Treaty News, n³, vol.2, avril 2012.

Florian Grisel, « Arbitrage d'investissement et promotion du développement économique: une étude de cas », dans OECD Global Forum on International Investment VII, 27-28 March 2008.

Lukombe Nghenda, Zaïrianisation, radicalisation et rétrocession en République du Zaïre. Considérations juridiques, Kinshasa, 1979,

Ludo Martens, Kabila et la révolution congolaise. Panafricanisme ou néocolonialisme?, Tome 1, Anvers, 2002. 
La protection internationale des investissements en République démocratique du Congo à l'aune de l'affaire Patrick Mitchell devant le CIRDI

Andrew Newcombe and Lluis Paradell, Law and Practice of Investment Treaties, Austin, Boston, Chicago, New York, The Netherlands,, 2009.

Nguyen Quoc Dihn, Patrick Daillier, Mathias Forteau, Alain Pellet, Droit international public, $8^{\text {ème }}$ édition, Paris, 2009. 